Clinical Vistas

\section{Penetrating pharyngeal injury in an infant}

A I-year-old boy was brought to the emergency department because his parents were concerned that he might have ingested a pencil-tip eraser. His parents did not witness the event, but they reported that they had heard choking sounds for a few seconds, followed by I episode of o vomiting. The child had no history of dyspnea, and he appeared well after vomiting. Upon physical examination, the child was afebrile, and he did not show symptoms of severe infection or signs of respiratory distress. There was a small bruise on the patient's left ton- sil and a 2-mm laceration on his tonsillar pillar. Auscultation confirmed normal breath sounds.

A radiograph of the neck showed diffuse subcutaneous emphysema consistent with a puncture wound (Figure I), and a radiograph of the chest showed emphysematous collections of air along the fascial planes of the neck (Figure 2). A computed tomography scan (Figure 3) showed extensive emphysematous collections surrounding the carotids. The patient received a diagnosis of subcutaneous emphysema of the neck and pneumomediatinum secondary to an oropharynx puncture wound.

After consultation with an otolaryngologist, the patient was admitted to hospital and received empiric antibiotic therapy. The patient's condition was monitored by one-to-one nursing in case of airway compromise. The results of a contrast study using a water-soluble agent showed no active perforation of the esophagus. Our patient was discharged 3 days after admission. At a 6-month followup visit, there were no complications or concerns.

Although pharyngeal perforation is rare in children, perforation can result

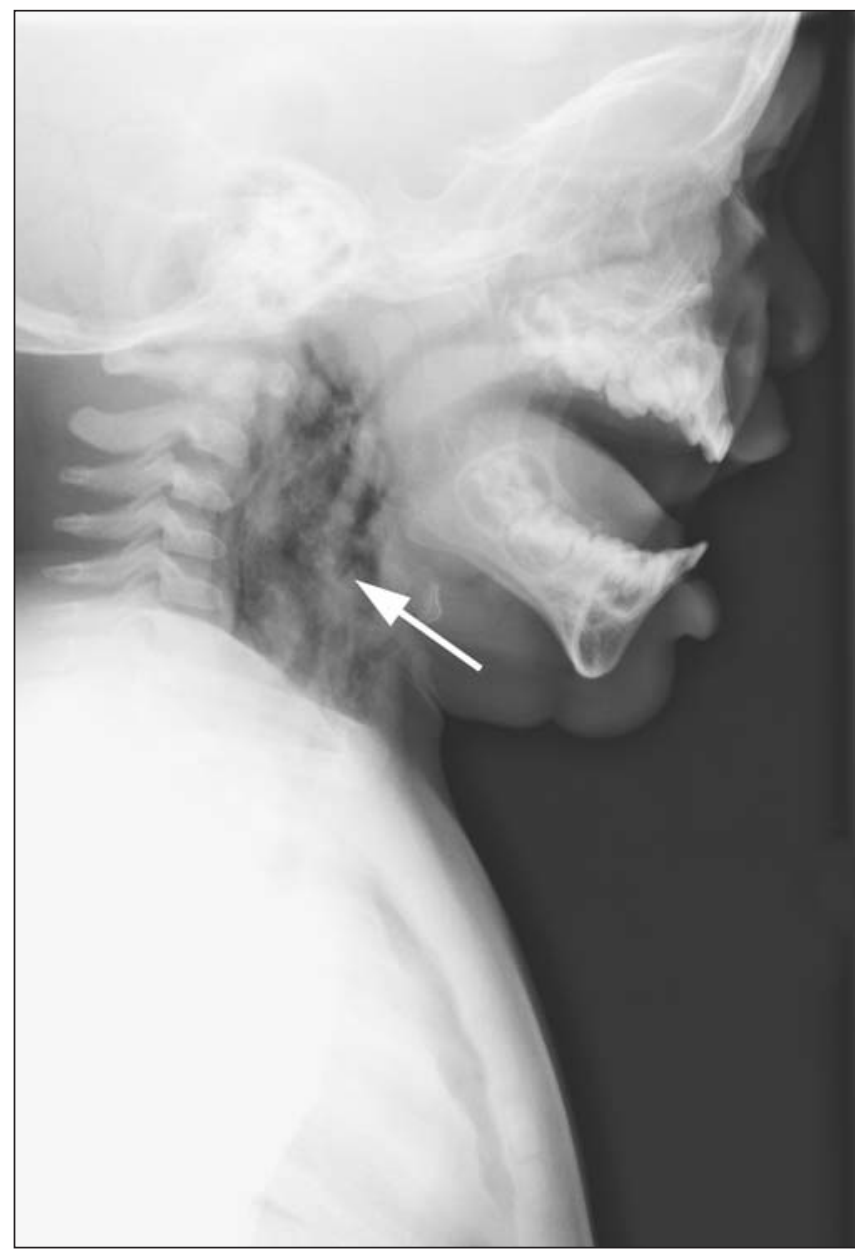

Figure 1: Neck radiograph showing diffuse subcutaneous emphysema (arrow) consistent with a puncture wound.

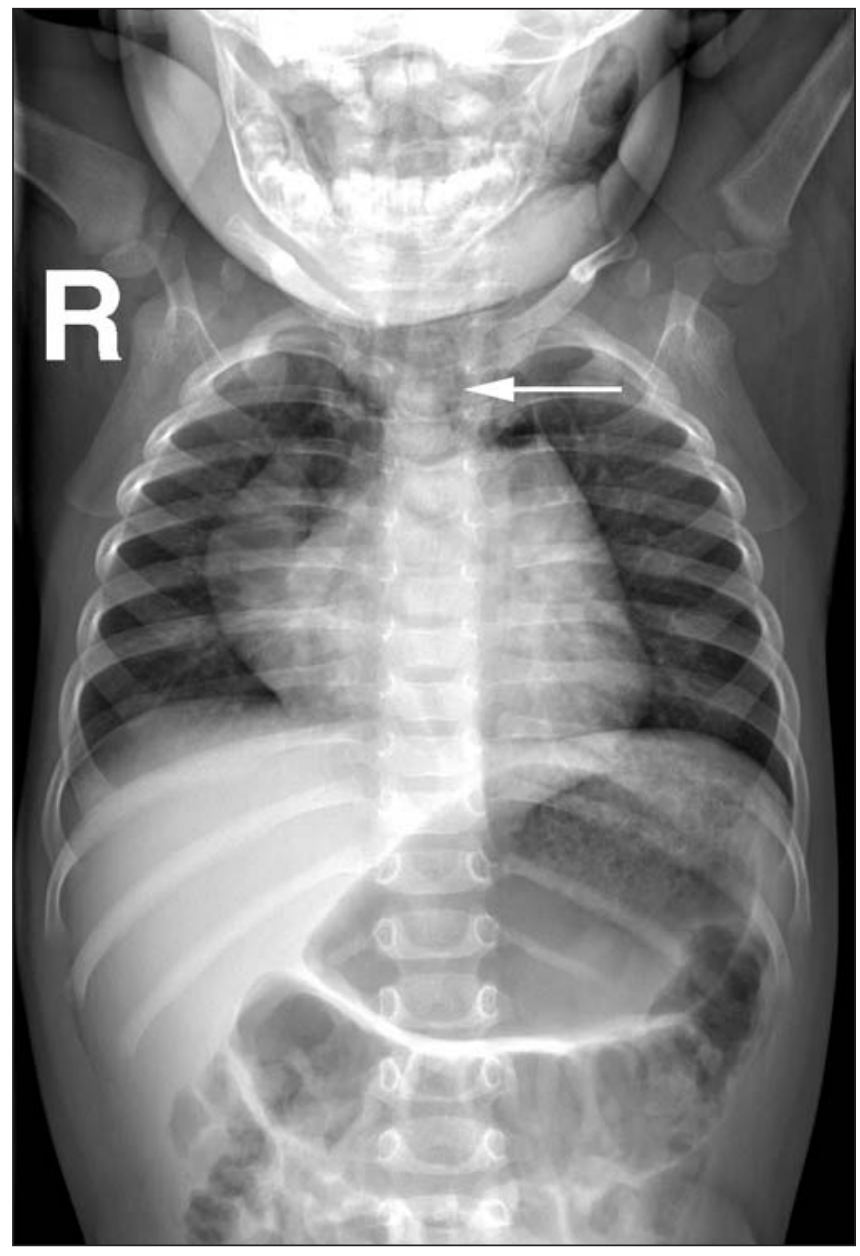

Figure 2: Chest radiograph showing emphysematous collections (arrow) along the fascial planes of the neck. 
when a child swallows a foreign object. ${ }^{1}$ Pharyngeal trauma can be associated with trivial injuries that are often

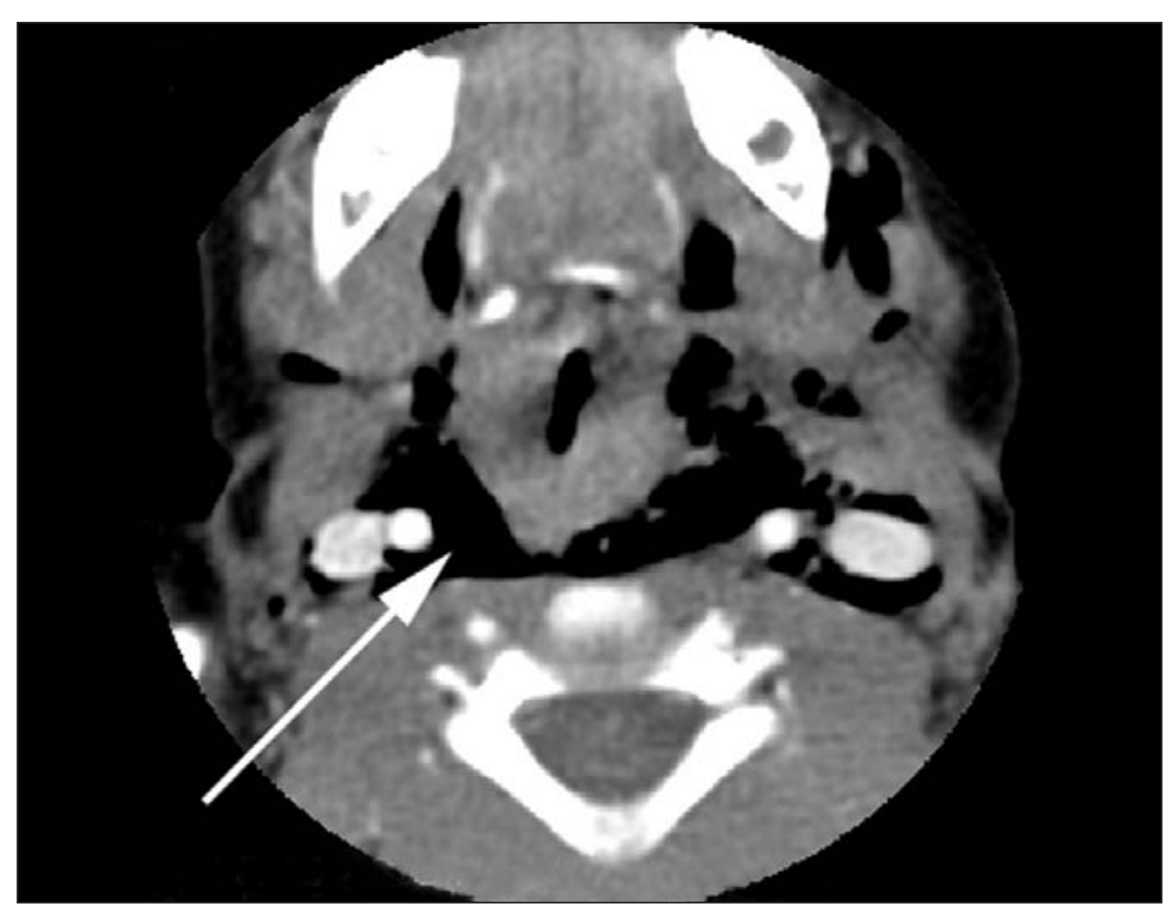

Figure 3: Computed tomography scan showing extensive emphysematous collections sur-

rounding the carotids (arrow).

unwitnessed, and the patients often present with incomplete histories. A tragic, but important, cause of pharyn- geal trauma is child abuse. ${ }^{2}$ Inconsistencies in the patient's history may alert clinicians to this serious problem. A delay in the recognition and management of pharyngeal trauma can lead to avoidable complications such as retropharyngeal abscess, mediastinitis and airway compromise.

\section{Rodrick Lim MD BSc}

Paediatric Emergency Department Children's Hospital of Western

Ontario

Michael Peddle MD BScH

Emergency Medicine

London Health Sciences Centre

London, Ont.

This article has been peer reviewed.

Competing interests: None declared.

\section{REFERENCES:}

I. Luqman Z, Khan MA, Nazir Z. Penetrating pharyngeal injuries in children: trivial trauma leading to devastating complications. Pediatr Surg Int 2005;2I:432-5.

2. Ramnarayan P, Qayyum A, Tolley N, et al. Subcutaneous emphysema of the neck in infancy: underrecognized presentation of child abuse. J Laryngol Otol 2004;II8:468-70.

\section{Public health}

\section{Autoimmune diseases after adolescent or adult}

\section{immunization: What should we expect?}

I n October 2006, the Israeli minister of health temporarily suspended the national influenza immunization program after 4 elderly patients with underlying cardiovascular disease suddenly died only days after receiving the influenza vaccine. There is compelling evidence that subunit and protein-based vaccines do not trigger sudden death $\rightarrow$ even in the most vulnerable infants ${ }^{1}$ and that influenza vaccines are safe for elderly patients. ${ }^{2}$ Despite this evidence and the absence of a biological hypothesis that connects injection of a subunit vaccine with sudden death, the psychologic impact of the close timing between in- jection and death was so strong that vaccination was considered as a potential death trigger. Although this was later proven not to be the case, ${ }^{3}$ this example illustrates how the rational approach of distinguishing a coincidence from a cause $e^{4}$ fades when a severe unexpected event occurs soon after what one might instinctively believe to be a putative cause.

Both human instinct and the art of medicine include the search for causes and triggers of adverse events. The intensity of the search increases when the outcome is unexpected, severe or disabling; when it is poorly understood; and when it affects a previously healthy person whom we love or care for. Accordingly, decades of experience and multiple large-scale epidemiologic studies have not yet convinced all people that vaccination does not cause sudden infant death, autism or asthma. The likelihood of an event being considered a trigger or a cause of disease apparently increases if the event is perceived to be aggressive (needle, compulsory immunization) or complex (immune stimulation), if it has longlasting effects (induction of immunity) or if the disease is only partly characterized. New vaccines meet all of these criteria; thus, the likelihood of a new vaccine being incriminated as a trigger for a severe outcome is extremely high.

The likelihood of a temporal association between vaccination and a given disease is proportional to vaccine coverage. 